\title{
Association between latent toxoplasmosis and major depression, generalised anxiety disorder and panic disorder in human adults
}

\author{
${ }^{1}$ Department of Psychology, Brigham Young University, Provo, Utah, USA; \\ ${ }^{2}$ The Neuroscience Center, Brigham Young University, Provo, Utah, USA; \\ ${ }^{3}$ Department of Sociology, Brigham Young University, Provo, Utah, USA
}

Shawn D. Gale ${ }^{1,2}$, Bruce L. Brown ${ }^{1}$, Andrew Berrett ${ }^{1}$, Lance D. Erickson ${ }^{3}$ and Dawson W. Hedges ${ }^{1,2}$

\begin{abstract}
Latent infection with the apicomplexan Toxoplasma gondii (Nicolle et Manceaux, 1908) has been associated with schizophrenia, bipolar disorder and self-harm behaviour. However, the potential relationship between T. gondii immunoglobulin G antibody (IgG) seropositivity and generalised-anxiety disorder (GAD) and panic disorder (PD) has not been investigated. The associations between serum reactivity to T. gondii and major depressive disorder (MDD), GAD and PD were evaluated in a total sample of 1846 adult participants between the ages of 20 and 39 years from the United States Center for Disease Control's National Health and Nutrition Examination Survey (NHANES). Approximately 16\% of the overall sample was seropositive for T. gondii and 7\% of the sample met criteria for MDD, $2 \%$ for GAD and $2 \%$ for PD. There were no significant associations between T. gondii IgG seroprevalence and $\mathrm{MDD}(\mathrm{OR}=0.484,95 \% \mathrm{CI}=0.186-1.258), \mathrm{GAD}(\mathrm{OR}=0.737,95 \% \mathrm{CI}=0.218-2.490)$ or $\mathrm{PD}(\mathrm{OR}=0.683,95 \%$ $\mathrm{CI}=0.206-2.270)$ controlling for sex, ethnicity, poverty-to-income ratio and educational attainment. However, limited evidence suggested a possible association between absolute antibody titres for T. gondii and GAD and PD but not MDD. Toxoplasma gondii seroprevalence was not associated with MDD, GAD or PD within the context of the limitations of this study, although there may be an association of $T$. gondii serointensity with and GAD and PD, which requires further study.
\end{abstract}

Keywords: Toxoplasma gondii, behaviour, mood, psychiatric, National Health and Nutrition Examination Survey (NHANES)

Toxoplasma gondii (Nicolle et Manceaux, 1908) is an intracellular apicomplexan parasite (Berenreiterová et al. 2011) that can infect humans via congenital transmission or ingestion of either tissue cysts from undercooked meat or oocysts from cat faeces (Carruthers and Suzuki 2007). Ingestion of raw oysters, clams and mussels or other raw or undercooked meats such as pork and lamb has also been linked with human $T$. gondii infection (Jones et al. 2009). Felines, including domestic cats, are the definitive host of $T$. gondii, but humans and a variety of other vertebrates including rodents are intermediate hosts. In intermediate hosts following acute infection, $T$. gondii can enter a latent phase, in which cysts form in neurons, microglia and astrocytes and in muscles, where they appear to persist for the life of the host (Dubey 1998, Carruthers and Suzuki 2007, Berenreiterová et al. 2011).

The prevalence of seropositivity for immunoglobulin $\mathrm{G}$ antibodies (IgG) against $T$. gondii varies by age, ethnic group and region (Jones et al. 2003). The overall worldwide prevalence of IgG seropositivity for T. gondii is approximately 30\% (Montoya and Liesenfeld 2004, Henriquez et al. 2009).

In an immunocompetent host, latent infection with T. gondii had been considered to be asymptomatic (Car- ruthers and Suzuki 2007). However, based on the affinity of $T$. gondii for the brain, there has been interest in how latent toxoplasmosis diagnosed by $\mathrm{IgG}$ antibodies against $T$. gondii may affect brain function and behaviour. In animal models, latent toxoplasmosis has been associated with decreased learning and memory and increased activity (Xiao et al. 2012). Latent toxoplasmosis infection in rodents has been further associated with decreased avoidance of a novel object or odour (Webster 2007), and rodents with latent toxoplasmosis show a decreased avoidance response to feline odours potentially related to modification of the neural substrates underlying the anxiety and fear response (Vyas et al. 2007).

The decreased avoidance response may be sex-specific and many of the alterations in genetic expression and behavioural changes associated with latent toxoplasmosis appear to be sex-dependent in rodents (Xiao et al. 2012). Xiao et al. (2012) found that female mice with latent toxoplasmosis were attracted to cat odour and had motor hyperactivity in an open-field test, whereas male mice with latent toxoplasmosis showed deficits in socially transmitted food preference and odour perception. Berdoy et al. (2000) suggested the decreased perception of risk might be due to $T$. gondii acting as an anxiolytic-like agent. To- 
gether, these findings from animal models suggest that latent infection with toxoplasmosis may reduce an animal's avoidance to situations that normally would be avoided.

In humans, numerous behavioural changes are also associated with seropositivity for $T$. gondii. Latent infection with toxoplasmosis is associated with differences in temperament, decreased novelty seeking (Novotná et al. 2005, Skallová et al. 2005, Flegr 2013), and decreased conscientiousness. Flegr (2010) found that latent toxoplasmosis infection is associated with the self-reported perception of decreased diplomacy in both men and women. Flegr (2010) also reported that women with latent toxoplasmosis infection were not anxious in situations in which other people would be, a finding suggesting that latent toxoplasmosis could be anxiolytic. There is also a strong association between latent $T$. gondii infection and schizophrenia (Torrey et al. 2007, Fekadu et al. 2010) that may involve Toxoplasma-induced changes in brain dopamine (Flegr 2013). In addition to findings of psychiatric illness and differences in personality, a longitudinal study by Pedersen et al. (2012) found that women with latent toxoplasmosis had a higher risk of self-harm. The results of a cross-sectional study indicated that $T$. gondii seropositivity was associated with nonfatal suicidal violence (Zhang et al. 2012), findings that suggest seropositivity for $T$. gondii may be associated with disinhibition. Despite the apparent association between latent toxoplasmosis and disinhibition, fear and novelty seeking in animal models and human studies, few studies have examined whether latent toxoplasmosis in humans is associated with anxiety disorders such as panic disorder (PD) and generalised anxiety disorder (GAD), and with mood disorders such as major depressive disorder (MDD) (Fekadu et al. 2010). Two small studies involving primarily subjects with schizophrenia found no association between latent infection with toxoplasmosis and MDD (Cetinkaya et al. 2007, Hamidinejat et al. 2010), but another study, while finding no difference in the prevalence of latent infection with $T$. gondii between a major-depression group and controls, did find more people with high antibody titres against $T$. gondii in the group with depression than in the control group (Hinze-Selch et al. 2007). Based on data from the Third National Health and Nutrition Examination Survey (NHANES), which collected data from 1988 to 1994, Pearce et al. (2012) found an association between latent infection with $T$. gondii and bipolar I disorder but no association with a history of major depression, severe major depression, or major depression comorbid with dysthymia as diagnosed by the Diagnostic Interview Schedule (DIS) (Helzer and Robins 1988). In a case-control study in a French sample, latent toxoplasmosis was associated with bipolar disorder (odds ratio 2.17, $p=0.028$ ) (Hamdani et al. 2013).

To our knowledge, no study to date has investigated the association between latent infection with $T$. gondii and either GAD or PD. To further clarify the association between unipolar mood disorders and latent toxoplasmosis and to investigate the relationship between latent toxoplasmosis and GAD and PD, we used data from the most recent continuous National Health and Nutrition Examination Surveys (NHANES) that contained the relevant data to determine whether latent toxoplasmosis identified by IGg antibody titre is associated with MDD, PD or GAD in a large community sample weighted to be representative of the United States population.

\section{MATERIALS AND METHODS}

\section{Study sample}

We obtained cross-sectional data about MDD, GAD and PD, Toxoplasma gondii IgG seropositivity, ethnic background, sex, educational attainment, age and poverty-to-income ratio in adults from the Center for Disease Control and Prevention's continuous NHANES datasets. This dataset uses complex, multi-stage sampling requiring weighting to represent the non-institutionalised United States population, and the NHANES survey includes interviews, physical examinations and laboratory samples regarding participants' medical and nutritional status (CDC 2014).

In the present study, we include only those adults over age 20 years for whom all testing data, including information about the presence or absence of MDD, GAD and PD, anti-toxoplasmosis IgG antibody titres, and the relevant demographic and socioeconomic variables, were available. The NHANES dataset is acquired in different cross-sectional samples in different years. To increase the sample size and to take advantage of all the relevant NHANES data, we combined the 1999-2000, 2001-2002 and 2003-2004 NHANES datasets.

Because the NHANES survey collects different data points during different years, and because we excluded participants without all of the necessary data points, the final dataset only included men and women between the ages of 20 and 39 years of age. The combined data sets consisted of 31126 participants, of whom 1846 had all of the variables necessary for our study and were included in the analysis. In some analyses, somewhat more subjects had all of the necessary variables for that particular analysis than another, and so the sample size in some analyses varied.

The final sample size ranged up to 1846 , although this number varied between analyses because of differences in the numbers of subjects providing data relevant for the individual analyses; 1846 subjects had data for all of the variables used in the analyses. Of these 1846 subjects, the majority was NonHispanic White (50.43\%). Fifty-five per cent of this sample was female, and $49.19 \%$ had an education level greater than a highschool diploma (Table 1). The mean age for this study sample was $29.7 \pm 0.4$ years, and the mean poverty income ratio (PIR) was $2.31 \pm 0.88$ (Table 2).

\section{Toxoplasma gondii IgG antibody}

According to the NHANES lab methodology descriptions (CDC 2014), the presence and quantity of IgG antibodies to $T$. gondii were determined by enzyme-linked immunosorbent assay (ELISA). Using optical density readings from positive control sera wells, standard curves were constructed and readings were calibrated to World Health Organization (WHO) Toxo 60 serum. We used the NHANES guidelines for each dataset to de- 
Table 1. Frequency counts for the cross-classification of gender, ethnicity and education.

\begin{tabular}{lcccc}
\hline & $\begin{array}{c}\text { Less than } \\
\text { high school }\end{array}$ & $\begin{array}{c}\text { High school } \\
\text { diploma }\end{array}$ & $\begin{array}{c}\text { More than } \\
\text { high school }\end{array}$ & Totals \\
\hline Male & & & & \\
Mexican American & 127 & 64 & 55 & 246 \\
Non-Hispanic black & 55 & 53 & 75 & 183 \\
Non-Hispanic white & 44 & 121 & 233 & 398 \\
Total - male & 226 & 238 & 363 & 827 \\
Female & & & & \\
Mexican American & 131 & 61 & 75 & 267 \\
Non-Hispanic black & 52 & 52 & 115 & 219 \\
Non-Hispanic white & 57 & 121 & 355 & 533 \\
Total - female & 240 & 234 & 545 & 1019 \\
Totals & 466 & 472 & 908 & 1846 \\
\hline
\end{tabular}

termine anti-Toxoplasma IgG concentrations indicative of latent T. gondii infection and used a binary system to classify subjects according to whether they had latent infection with $T$. gondii.

In this way, we were able to set specific conditions for each individual data set, resulting in equivalent data across all three data sets and consistent with the NHANES guidelines for determining anti-Toxoplasma IgG seropositivity. Because HinzeSelch et al. (2007) reported an association between serointensity of anti-toxoplasmosis IgG and MDD, we also used absolute anti-Toxoplasma IgG antibody levels in additional analyses to determine the association between anti-Toxoplasma IgG antibody serointensity and MDD, PD, and GAD.

\section{Mood and Anxiety Disorders}

The NHANES survey used the World Health Organization's Composite International Diagnostic Interview (CIDI), a comprehensive structured interview to evaluate and diagnose mental disorders present in the past 12 months. The CIDI is based on diagnostic criteria according to the tenth revision of the International Classification of Diseases and the fourth edition of the Diagnostic and Statistical Manual of Mental Disorders. Specifically, NHANES did not include the diagnostic modules for all mental disorders addressed in the CIDI; rather, it utilised the diagnostic modules for the diagnoses of MDD, PD and GAD.

\section{Control variables}

Demographic data were collected for all survey participants through a household interview prior to the health examination. The NHANES computer-assisted personal interview software program was used to collect and record the interview data (CDC 2014). Since the prevalence of latent infection with T. gondii can vary by age, ethnic group and region, we adjusted for the following potentially confounding variables: gender, age, education (less than high school, high-school diploma, more than high school), ethnicity (Mexican American, Non-Hispanic white, Non-Hispanic black, and Other), and poverty income ratio (PIR), a measure of assessing household wealth (total family income divided by the United States poverty threshold adjusted for family size) with a value less than 1 indicative of an income below the poverty threshold.

To produce unbiased national estimates, weights were created for each two-year data set. We obtained the weights used in our study from the mood-disorder data sets in the examination portion of the NHANES since they were completely nested within both the T. gondii and demographics data sets. A set of
Table 2. Mean \pm standard deviation of ages and poverty-to-income ratio (PIR) for the cross-classification of gender, ethnicity and education.

\begin{tabular}{lcccc}
\hline & $\begin{array}{c}\text { Less than } \\
\text { High school }\end{array}$ & $\begin{array}{c}\text { High school } \\
\text { diploma }\end{array}$ & $\begin{array}{c}\text { More than } \\
\text { high school }\end{array}$ & Totals \\
\hline Male-Age & & & & \\
Mexican American & $29.2 \pm 5.8$ & $29.6 \pm 5.7$ & $30.0 \pm 5.0$ & $29.6 \pm 5.5$ \\
Non-Hispanic black & $30.0 \pm 6.1$ & $29.8 \pm 6.1$ & $29.4 \pm 5.9$ & $29.7 \pm 6.0$ \\
Non-Hispanic white & $30.3 \pm 6.6$ & $30.3 \pm 6.0$ & $30.1 \pm 5.5$ & $30.2 \pm 6.0$ \\
Total- male & $29.6 \pm 6.0$ & $30.0 \pm 5.9$ & $29.9 \pm 5.5$ & $29.8 \pm 5.8$ \\
Female-Age & & & & \\
Mexican American & $28.3 \pm 5.5$ & $28.9 \pm 5.8$ & $30.1 \pm 5.9$ & $29.1 \pm 5.7$ \\
Non-Hispanic black & $31.6 \pm 6.2$ & $29.8 \pm 6.4$ & $30.0 \pm 5.3$ & $30.5 \pm 6.0$ \\
Non-Hispanic white & $28.7 \pm 6.3$ & $29.4 \pm 6$. & $30.5 \pm 5.2$ & $29.5 \pm 5.9$ \\
Total- female & $29.1 \pm 6.0$ & $29.4 \pm 6.2$ & $30.3 \pm 5.3$ & $29.6 \pm 5.8$ \\
Totals -Age & $29.3 \pm 6.1$ & $29.7 \pm 6.1$ & $30.1 \pm 5.5$ & $29.7 \pm 5.8$ \\
Male-PIR & & & & \\
Mexican American & $1.52 \pm 1.01$ & $2.13 \pm 1.25$ & $2.78 \pm 1.50$ & $2.14 \pm 1.25$ \\
Non-Hispanic black & $1.60 \pm 1.26$ & $2.31 \pm 1.38$ & $2.71 \pm 1.62$ & $2.21 \pm 1.42$ \\
Non-Hispanic white & $1.83 \pm 1.23$ & $2.67 \pm 1.48$ & $3.62 \pm 1.55$ & $2.70 \pm 1.42$ \\
Total- male & $1.69 \pm 1.12$ & $2.44 \pm 1.41$ & $3.30 \pm 1.61$ & $2.45 \pm 1.38$ \\
Female-PIR & & & & \\
Mexican American & $1.20 \pm 0.81$ & $1.79 \pm 1.30$ & $2.64 \pm 1.63$ & $1.87 \pm 1.25$ \\
Non-Hispanic black & $1.11 \pm 1.10$ & $1.61 \pm 1.30$ & $2.40 \pm 1.48$ & $1.71 \pm 1.29$ \\
Non-Hispanic white & $1.78 \pm 1.57$ & $2.41 \pm 1.45$ & $3.45 \pm 1.51$ & $2.55 \pm 1.51$ \\
Total- female & $1.32 \pm 1.12$ & $2.07 \pm 1.42$ & $3.12 \pm 1.59$ & $2.17 \pm 1.38$ \\
Totals - PIR & $1.46 \pm 1.15$ & $2.26 \pm 1.37$ & $3.21 \pm 1.56$ & $2.31 \pm 1.36$ \\
\hline & & & &
\end{tabular}

four-year weights had been created by the Center for Disease Control for the 1999-2000 and 2001-2002 data sets. We used the four-year weights for the first two data sets and a two-year weight created for the 2003-2004 data set (CDC 2005, 2014). In order to control for sampling-errors, stratum and primary sampling units were used. These variables were included in, and obtained from, the demographic data sets.

\section{Statistical analyses}

The three two-year data sets were appended by hand using Microsoft Excel 2013, where we were also able to set specific stipulations to our data such as the IgG-titre thresholds. Variables of interest were combined into one data set through the use of the SAS MERGE function (SAS Institute, Cary, North Carolina). Logistic regression models were estimated in Stata 13.1 (Stata Corp. 2013). In our analyses, poverty-to-income ratio (PIR) and age were quantitative, whereas latent Toxoplasma infection, depression and anxiety disorders (e.g. MDD, PD, GAD), education, gender and ethnicity were all categorical variables. We also estimated analyses with anti-toxoplasmosis $\operatorname{IgG}$ antibody level as a continuous variable separately for latent Toxoplasma-positive and negative groups. Analyses were performed with and without covariates to assess possible confounding.

\section{RESULTS}

Of the sample involving 1846 participants, $15.8 \%$ were seropositive for $T$. gondii IgG antibodies. There was a slightly higher prevalence of infection in males (17.4\%) compared to females $(13.6 \%)$, a difference that was not statistically significant, $\left(X^{2}(1)=2.378, p=0.123\right)$. Toxoplasmosis seroprevalence differed by education level: less 
Table 3. Logistic regressions predicting major depressive disorder, panic disorder and generalised anxiety disorder from Toxoplasma gondii IgG, gender, age, education level, poverty-to-income ratio (PIR) and ethnicity.

\begin{tabular}{|c|c|c|c|c|c|c|c|c|c|c|c|c|}
\hline \multicolumn{13}{|c|}{ Major depressive disorder } \\
\hline & \multicolumn{4}{|c|}{ Males } & \multicolumn{4}{|c|}{ Females } & \multicolumn{4}{|c|}{ Full sample } \\
\hline & $\beta$ & OR & $95 \% \mathrm{CI}$ & $\mathrm{p}$ & $\beta$ & OR & $95 \% \mathrm{CI}$ & $\mathrm{p}$ & $\beta$ & OR & $95 \% \mathrm{CI}$ & $\mathrm{p}$ \\
\hline Toxoplasma positive & -.720 & .487 & {$[.161,1.471]$} & .196 & -.745 & .475 & {$[.107,2.113]$} & .320 & -.726 & .484 & {$[.107,2.184]$} & .337 \\
\hline Male $^{\mathrm{a}}$ & - & - & - & - & - & - & - & - & -.403 & .669 & {$[.431,1.037]$} & .071 \\
\hline Toxoplasma $\mathrm{X} \mathrm{male}^{\mathrm{a}, \mathrm{b}}$ & - & - & - & - & - & - & - & - & -.001 & .999 & {$[.162,6.148]$} & .999 \\
\hline Age & .005 & 1.005 & {$[1.001,1.009]$} & .026 & .003 & 1.003 & {$[.998,1.007]$} & .214 & .003 & 1.003 & {$[1.000,1.007]$} & .033 \\
\hline \multicolumn{13}{|l|}{ Race-ethnicity } \\
\hline Non-Hispanic white & .000 & 1.000 & {$[1.000,1.000]$} & - & .000 & 1.000 & {$[1.000,1.000]$} & - & .000 & 1.000 & {$[1.000,1.000]$} & - \\
\hline Non-Hispanic black & -1.189 & .305 & {$[.097, .959]$} & .043 & -1.073 & .342 & {$[.147, .797]$} & .014 & -1.118 & .327 & {$[.195, .547]$} & .000 \\
\hline Hispanic & -.126 & .882 & {$[.400,1.945]$} & .750 & -.922 & .398 & {$[.166, .950]$} & .038 & -.595 & .551 & {$[.327, .929]$} & .026 \\
\hline Education & -.319 & .727 & {$[.387,1.363]$} & .312 & -.247 & .781 & {$[.539,1.133]$} & .188 & -.269 & .764 & {$[.564,1.035]$} & .081 \\
\hline Poverty-to-income ratio & .058 & 1.060 & {$[.869,1.293]$} & .556 & -.209 & .811 & {$[.638,1.031]$} & .085 & -.098 & .906 & {$[.779,1.054]$} & .196 \\
\hline Constant $^{\mathrm{c}}$ & -3.627 & - & {$[-5.569,-1.686]$} & - & -1.900 & - & {$[-3.607,-.193]$} & - & -2.404 & - & {$[-3.812,-.996]$} & - \\
\hline $\mathrm{N}$ & & & 824 & & & & 1019 & & & & 1843 & \\
\hline \multicolumn{13}{|c|}{ Panic disorder (PD) } \\
\hline & \multicolumn{4}{|c|}{ Males } & \multicolumn{4}{|c|}{ Females } & \multicolumn{4}{|c|}{ Full sample } \\
\hline & $\beta$ & OR & $95 \% \mathrm{CI}$ & $\mathrm{p}$ & $\beta$ & OR & $95 \% \mathrm{CI}$ & $\mathrm{p}$ & $\beta$ & OR & $95 \% \mathrm{CI}$ & $\mathrm{p}$ \\
\hline positive $^{\mathrm{d}}$ & - & - & - & - & .240 & 1.271 & {$[.366,4.409]$} & .700 & -.381 & .683 & {$[.199,2.343]$} & .537 \\
\hline Male $^{\mathrm{a}}$ & - & - & - & - & - & - & - & - & -.476 & .622 & {$[.294,1.313]$} & .207 \\
\hline Toxoplasma $\mathrm{X} \mathrm{male}^{\mathrm{a}, \mathrm{b}}$ & - & - & - & - & - & - & - & - & - & - & - & - \\
\hline Age & -.003 & .997 & {$[.987,1.007]$} & .570 & .004 & 1.004 & {$[.998,1.010]$} & .156 & .002 & 1.002 & {$[.997,1.007]$} & .472 \\
\hline \multicolumn{13}{|l|}{ Race-ethnicity } \\
\hline Non-Hispanic white & .000 & 1.000 & {$[1.000,1$} & - & .00 & 1.000 & {$[1.000,1$} & - & .000 & 1.000 & {$[1$.} & - \\
\hline Non- & -.382 & .682 & {$[.157,2$. } & .602 & -2.500 & .082 & {$[.032$,} & .000 & -1.467 & .231 & {$[.082,$.} & .006 \\
\hline Hispa & -.632 & .531 & {$[.059,4.7$} & .565 & -1.059 & .347 & {$[.134, .896]$} & .030 & -.855 & .425 & {$[.174,1.039]$} & .060 \\
\hline Education & -.274 & .760 & {$[.318,1.816]$} & .529 & -.421 & .657 & {$[.398,1.083]$} & .098 & -.382 & .682 & {$[.453,1.028]$} & .067 \\
\hline Poverty-to-income ratio & -.242 & .785 & {$[.562,1.097]$} & .151 & -.712 & .490 & {$[.355, .678]$} & .000 & -.519 & .595 & {$[.448, .791]$} & .001 \\
\hline Constant $^{\mathrm{c}}$ & -1.546 & - & {$[-6.282,3.190]$} & - & -2.378 & - & {$[-4.934, .178]$} & - & -1.877 & - & {$[-4.317, .563]$} & - \\
\hline $\mathrm{N}$ & & & 684 & & & & 1019 & & & & 1846 & \\
\hline \multicolumn{13}{|c|}{ Generalised anxiety disorder (GAD) } \\
\hline & \multicolumn{4}{|c|}{ Males } & \multicolumn{4}{|c|}{ Females } & \multicolumn{4}{|c|}{ Full sample } \\
\hline & $\beta$ & OR & $95 \% \mathrm{CI}$ & $\mathrm{p}$ & $\beta$ & OR & $95 \% \mathrm{CI}$ & $\mathrm{p}$ & $\beta$ & OR & $95 \% \mathrm{CI}$ & $\mathrm{p}$ \\
\hline Toxoplasma positive $\mathrm{d}^{\mathrm{d}}$ & - & - & - & - & .402 & 1.494 & {$[.437,5.113]$} & .514 & -.306 & .737 & {$[.211,2.571]$} & .625 \\
\hline Male $^{\mathrm{a}}$ & - & - & - & - & - & - & - & - & -.368 & .692 & {$[.293,1.638]$} & .394 \\
\hline Toxoplasma $\mathrm{X}$ male $\mathrm{e}^{\mathrm{a}, \mathrm{b}}$ & - & - & - & - & - & - & - & - & - & - & - & - \\
\hline Age & .006 & 1.006 & {$[.993,1.019]$} & .374 & .006 & 1.006 & {$[.999,1.012]$} & .074 & .006 & 1.006 & {$[1.000,1.012]$} & .060 \\
\hline \multicolumn{13}{|l|}{ Race-ethnicity } \\
\hline Non-Hispanic white & .000 & 1.000 & {$[1.000,1.000]$} & - & .000 & 1.000 & {$[1.000,1.000]$} & - & .000 & 1.000 & {$[1.000,1.000]$} & - \\
\hline Non-Hispanic black & -.458 & .633 & {$[.186,2.153]$} & .455 & -2.243 & .106 & {$[.037, .302]$} & .000 & -1.323 & .266 & {$[.108, .654]$} & .005 \\
\hline Hispanic & -1.684 & .186 & {$[.021,1.637]$} & .126 & -.493 & .611 & {$[.202,1.843]$} & .373 & -.660 & .517 & {$[.208,1.286]$} & .152 \\
\hline Education & -.228 & .796 & {$[.197,3.223]$} & .744 & -.453 & .636 & {$[.356,1.137]$} & .123 & -.391 & .677 & {$[.395,1.160]$} & .151 \\
\hline Poverty-to-income ratio & -.404 & .668 & {$[.209,2.132]$} & .487 & -.714 & .490 & {$[.332, .723]$} & .001 & -.567 & .567 & {$[.356, .905]$} & .019 \\
\hline Constant $^{\mathrm{c}}$ & -4.343 & - & {$[-9.564, .878]$} & - & -3.149 & - & {$[-5.254,-1.043]$} & . & -3.435 & - & {$[-5.921,-.949]$} & - \\
\hline $\mathrm{N}$ & & & 684 & & & & 1019 & & & & 1846 & \\
\hline
\end{tabular}

$\mathrm{a}$ - gender is constant in male and female only models; $\mathrm{b}$ - interactions between Toxoplasma and gender could not be estimated for PD or GAD because there were no male subjects with latent toxoplasmosis and either disorder; $\mathrm{c}-95 \%$ confidence interval reported is for $\beta ; \mathrm{d}-$ Toxoplasma is constant in male only models for PD or GAD because there were no male subjects with latent toxoplasmosis and either disorder; source: NHANES 1999-2000, 2001-2002, and 2003-2004.

than high school (25.9\%), high school (16.1\%), and more than high school $(9.4 \%),\left(X^{2}(2)=47.708, p<0.0001\right)$. Seroprevalence by ethnicity was highest in those identifying themselves as Mexican Americans (23.2\%). The NonHispanic white group had the lowest prevalence at $10.3 \%$, and the Non-Hispanic black group had a prevalence of $16.7 \%$. The overall difference in prevalence between the three groups was statistically significant $\left(X^{2}(2)=21.760\right.$, $p<0.0001)$.

Approximately $7 \%$ of the sample met CIDI criteria for MDD, 2\% for GAD and 2\% for PD. Mexican Americans were significantly less likely than non-Hispanic whites to have MDD $(\mathrm{OR}=0.551,95 \% \mathrm{CI}=0.327-0.930)$ but not $\mathrm{PD}(\mathrm{OR}=0.425,95 \% \mathrm{CI}=0.174-1.039)$ or $\mathrm{GAD}$ 
$(\mathrm{OR}=0.517,95 \%=0.208-1.286)$. Males displayed a consistently lower prevalence among the three disorders, although the relationship was not statistically significant. Age was positively associated with depression $(\mathrm{OR}=1.003,95 \% \mathrm{CI}=1.000-1.007)$, although the age range of the entire sample was only from 20 to 39 years. Higher values of PIR were associated with fewer cases of both $\mathrm{PD}(\mathrm{OR}=0.595,95 \% \mathrm{CI}=0.448-0.791)$ and $\mathrm{GAD}$ $(\mathrm{OR}=0.567,95 \% \mathrm{CI}=0.356-0.905)$. Our results using presence or absence of latent toxoplasmosis showed no significant association in the fully adjusted model with covariates between $T$. gondii $\mathrm{IgG}$ seroprevalence and MDD $(\mathrm{OR}=0.484,95 \% \mathrm{CI}=0.181-1.290), \mathrm{PD}(\mathrm{OR}=0.683$, $95 \% \mathrm{CI}=0.199-2.343)$ or $\mathrm{GAD}(\mathrm{OR}=0.737,95 \%$ $\mathrm{CI}=0.211-2.571)$ (Table 3 ). We also estimated models that included interactions between $T$. gondii and gender but do not report them because the interactions were nonsignificant.

A logistic regression model with anti-toxoplasmosis IgG antibody as a continuous variable was carried out separately for the latent toxoplasmosis positive and negative groups to determine if there were differences in serointensity associated with MDD. It should be pointed out that the NHANES database arbitrarily recorded all IgG antibody values $\geq 240$ as 240 . Thus, the estimates of the relationship between serointensity reported here are likely conservative for the latent Toxoplasma-positive group as there were many participants with values of 240. In this logistic regression analysis, the IgG level was non-significantly related to MDD in both the latent Toxoplasma-positive $(\mathrm{OR}=1.0351,95 \% \mathrm{CI}=0.8570-1.2502, \mathrm{n}=1813)$ and negative group $(\mathrm{OR}=1.0002,95 \% \mathrm{CI}=0.9900-1.0105$, $\mathrm{n}=290)$ when modelled without control variables. In models controlling for gender, age, ethnic background, educational attainment and PIR, IgG antibody levels were again non-significant for the latent Toxoplasma-positive $(\mathrm{OR}=0.9971,95 \% \mathrm{CI}=0.7906-1.2576, \mathrm{n}=1562)$ and negative groups $(\mathrm{OR}=0.9996,95 \% \mathrm{CI}=0.9876-1.0118$, $\mathrm{n}=225$ ).

We also estimated logistic regression models with and without control variables for the relationship of anti-toxoplasmosis IgG antibodies serointensity with PD and GAD for latent Toxoplasma-positive and negative groups. Similar to the findings for MDD, the IgG levels were not associated with PD among the latent Toxoplas$m a$-negative group in a model without control variables $(\mathrm{OR}=0.9575,95 \% \mathrm{CI}=0.6737-1.3608, \mathrm{n}=1818)$ as well as a model with control variables $(\mathrm{OR}=0.9348$, $95 \% \mathrm{CI}=0.6006-1.4550, \mathrm{n}=1565)$. On the other hand, the relationship was statistically significant in the latent Toxoplasma-positive group ( $\mathrm{OR}=1.0223,95 \%$ $\mathrm{CI}=1.0030-1.0420, \mathrm{n}=290)$. However, after adding control variables, the relationship between IgG levels in PD was no longer significant $(\mathrm{OR}=1.0366,95 \%$ $\mathrm{CI}=0.9985-1.0760, \mathrm{n}=58) . \mathrm{IgG}$ levels were also not associated with GAD among the latent Toxoplasma-negative group without control variables $(\mathrm{OR}=0.9189,95 \%$ $\mathrm{CI}=0.6649-1.2697, \mathrm{n}=1818)$ or with control variables $(\mathrm{OR}=0.8171,95 \% \mathrm{CI}=0.5067-1.3176, \mathrm{n}=1565)$. Interestingly, models for PD and GAD were identical for the latent Toxoplasma-positive group. This was because, in every case, PD and GAD were comorbid. In other words, among respondents in the latent toxoplasmosis positive group, those who had PD also had GAD. Consequently, the results for PD described above also characterised the relationship between IgG levels and GAD.

\section{DISCUSSION}

In contrast to studies that reported an association between latent toxoplasmosis and schizophrenia, bipolar disorder and self-harm, we found no association using presence or absence of latent toxoplasmosis as a binary variable between latent toxoplasmosis and MDD, GAD and PD adjusted for gender, ethnic origin, age, educational attainment and PIR in a sample representative of the United States population. In the unadjusted model for the latent Toxoplasma-positive group, there was an association of anti-Toxoplasma IgG antibodies with GAD and PD, which was no longer apparent after adding the control variables, although the number of subjects dropped to only 58 in the fully adjusted model. Any possible association of latent toxoplasmosis with GAD and PD requires further study.

However, the overall lack of association we found between latent toxoplasmosis and GAD and PD is broadly consistent with previous research in animal models showing decreased novelty avoidance (Webster 2007) and anxiolytic-like behaviour (Berdoy et al. 2000), findings suggesting that latent toxoplasmosis in rodents is associated with an anxiolytic-like response. The lack of an association with GAD and PD is further consistent with previous reports that latent toxoplasmosis in humans is associated with a perception of decreased diplomacy (Flegr 2010) and with decreased conscientiousness.

Further, the finding that Toxoplasma seroprevalence is not associated with GAD or PD is consistent with the report that women seropositive for toxoplasmosis were not anxious in situations in which other women would be (Flegr 2010). Latent toxoplasmosis in humans has been associated with behaviour characterised by decreased inhibition, such as increased risk for self-harm (Pedersen et al. 2012), whereas a characteristic of anxiety is increased inhibition. Our findings that latent toxoplasmosis in humans is not associated with GAD or PD as such are consistent with both animal models and human studies that show decreased inhibition and increased impulsivity (Webster 2007, Pedersen et al. 2012).

The lack of an association between latent toxoplasmosis and MDD that we found is similar to findings from a study done in China reporting that patients with affec- 
tive disorder did not have elevated seroprevalence of toxoplasmosis compared to controls (Wang et al. 2006) and with other previous studies (Cetinkaya et al. 2007, HinzeSelch et al. 2007, Hamidinejat et al. 2010, Pearce et al. 2012). In contrast to findings that high IgG anti-toxoplasmosis antibody levels were positively associated with MDD (Hinze-Selch et al. 2007), we found no evidence supporting an association between anti-Toxoplasma IgG serointensity and MDD.

In the present study, we found an overall United States prevalence of $15.8 \%$ for latent toxoplasmosis, which is similar to, but somewhat lower than, the prevalence of $22.5 \%$ reported by Jones et al. (2001), though this may have been due to methodological differences including a much smaller sample size and smaller age range in the present study. The prevalence did not differ significantly between men and women, consistent with previous findings (Montoya and Liesenfeld 2004). However, there was a significant difference in prevalence by ethnic groups and by education level. We also found that approximately $7 \%$ of the sample met criteria for 12-month prevalence of MDD and $2 \%$ for GAD and for PD. These prevalences for MDD, GAD and PD are similar to those reported by Kessler et al. (2012) showing a 12-month prevalence among people ages 18 to 64 years of MDD of $9.3 \%$, of GAD of $2.9 \%$, and of PD of $3.1 \%$, suggesting that the estimates obtained from the CIDI in the NHANES survey approximate the estimates obtained in epidemiological surveys.

The genetic and neural mechanisms associated with latent toxoplasmosis and associated behavioural changes reported in previous studies are unclear, although several possibilities exist. In mice, latent toxoplasmosis is associated with increased dopamine, although not with changes in serotonin (Stibbs 1985). Similarly, latent toxoplasmosis may increase dopamine metabolism (Prandovszky et al. 2011). Finally, rats with latent toxoplasmosis treated with a dopamine-2 antagonist decreased their risky behaviour (Webster et al. 2006), although the dopamine-2 antagonist haloperidol inhibits Toxoplasma growth in vitro (Jones-Brando 2003) and thus may have affected the animal behaviour not by antagonising dopamine but by inhibiting the infection itself.

Another possible mechanism underlying the behavioural changes associated with latent toxoplasmosis in previous studies has to do with the presence and distribution of parasitic cysts in the central nervous system. In mice, Berenreiterová et al. (2011) found that while latent toxoplasmosis cysts were distributed throughout the brain, the highest densities were in the telencephalon, hippocampus and amygdala with relative sparing of the cerebellum, caudate and putamen. Thus, based on the animal data, it would seem possible that parasitic cysts in these regions in humans could alter both frontal and limbic areas, as well as frontolimbic connectivity, which could then result in behavioural and emotional changes. Along these lines, prior work in humans has demonstrated an association between regional activity of the prefrontal cortex and amygdala with anxiety disorders (Etkin and Wager 2007) as well as decreased structural connectivity between these same regions (Tromp et al. 2012). However, abnormalities in these regions, particularly after an acquired lesion, have not always resulted in increased anxiety or depression. For example, decreases in emotional responsiveness and anxiety have also been demonstrated (Bechara et al. 2000, Vasa et al. 2004). As such, our findings suggesting no association between anti- $T$. gondii antibody seroprevalence and serointensity are consistent with the notion that frontal dysfunction does not always result in depression or anxiety.

In addition to neurotransmitter changes and cysticrelated structural changes in the brain (i.e. brain injury) associated with latent toxoplasmosis in animal models, there is also evidence for alterations in gene expression. Xiao et al. (2012) demonstrated that female mice infected with latent toxoplasmosis had altered gene expression associated with forebrain development and neurogenesis. Thus, cortical regions might not only be injured by the presence of cysts, but latent toxoplasmosis may alter the development of these same regions, which may help explain behaviours such as the locomotor hyperactivity females demonstrate in open field-testing. Accordingly, animal models suggest that latent toxoplasmosis has an affinity for cortical and select subcortical regions related to higher-order behaviour and impulse control as well as to limbic regions and emotional tone and regulation. Furthermore, alterations in neurotransmitter levels and metabolism and even gene expression of both neurotransmitters and forebrain development may also be altered.

Berenreiterová et al. (2011) suggest that latent toxoplasmosis may cause neuronal degeneration in local brain regions or produce an immune response that alters neurotransmitters, further mechanisms that could be associated with the behavioural changes associated with latent toxoplasmosis. Latent toxoplasmosis is associated with an immune response (Carruthers and Suzuki 2007) that could also alter brain function. In addition, host factors are likely important in determining susceptibility to toxoplasmosis (Carruthers and Suzuki 2007). In fact, in their study of toxoplasmosis cyst distribution in mice, Berenreiterová et al. (2011) found large interindividual variation in parasite load, indicating that individual host factors affect the parasite dose in an individual brain.

Strengths of the present study include the use of a large community sample with data combined over three NHANES surveys, in which we corroborate the findings of Pearce et al. (2012) suggesting that there is no association between seroprevalence of latent toxoplasmosis and MDD. The findings also provide limited support suggesting that anti-T. gondii antibody serointensity may be positively associated with GAD and PD. We also controlled 
for ethnic background, gender, socioeconomic status and education level. Further, to our knowledge this is the first study that has systematically evaluated the association between latent toxoplasmosis and PD and GAD.

Several limitations require consideration when interpreting our findings. The present study carries with it all the limitations associated with a cross-sectional design. Similarly, we simply used IgG antibody titre against $T$. gondii to determine seroprevalence of latent infection with toxoplasmosis and could not determine when the infection originally occurred. It is possible that length of time with a chronic infection may influence the risk of developing MDD, GAD or PD, or perhaps even symptom severity. Further, the diagnosis of major depressive disorder, generalised anxiety disorder and panic disorder relied exclusively on a computer-based structured interview completed by the subjects. There was no other diagnostic process such as a clinical interview by which to corroborate the questionnaire findings. Also, the CIDI was used to address the presence of these diagnoses in the past 12 months only. Since the NHANES dataset was not designed explicitly to evaluate the association between latent toxoplasmosis and MDD, GAD and PD, we were unable to use outcome data designed specifically for the question we investigated. While we attempted to adjust for ethnic background, age, gender, education and PIR as a proxy for socioeconomic status, other variables for which we did not control could have resulted in residual confounding. Finally, these particular NHANES data sets only allowed for inclusion of subjects whose ages were between 20 and 39 years. It is possible that subjects with a longer duration of Toxoplasma seropositivity could have had more depression and anxiety or perhaps risk of developing depression or anxiety may be linked to age at the time of initial acute infection. These specific possibilities could not be investigated in these cross-sectional NHANES data sets.

Although latent toxoplasmosis in humans has been associated with a variety of behavioural changes including self-inflicted aggression, bipolar disorder and schizophrenia, we found no evidence that it is associated with MDD within the context of the limitations of this study. More detailed assessment of mood state and affective regulation, however, could show evidence that latent toxoplasmosis affects mood. Further, we found that seroprevalence of latent toxoplasmosis was not associated with GAD or PD, although there was limited evidence of an association of IgG serointensity with GAD and PD, a possibility that requires further investigation. Given the high global prevalence of latent toxoplasmosis and evidence that it affects human behaviour and neuropsychological function, additional research into the neuropsychiatric and neuropsychological effects of latent toxoplasmosis is needed.

\section{REFERENCES}

Bechara A., Damasio H., Damasio A.R. 2000: Emotion, decision making and the orbitofrontal cortex. Cereb. Cortex. 10: 295-307.

Berdoy M., Webster J.P., Macdonald D.W. 2000: Fatal attraction in rats infected with Toxoplasma gondii. Proc. Biol. Sci. 267: 1591-1594.

Berenreiterová M., Flegr J., Kuběna A.A., Němec P. 2011: The distribution of Toxoplasma gondii cysts in the brain of a mouse with latent toxoplasmosis: implications for the behavioral manipulation hypothesis. PLoS ONE 6: e28925.

Carruthers V.B., Suzuki Y. 2007: Effects of Toxoplasma gondii infection on the brain. Schizophr. Bull. 33: 745-751.

CDC (Centers for Disease Control) 2005: Analytic and Reporting Guidelines: The National Health and Nutrition Examination Survey [Online]. U.S. Department of Health and Human Services. World Wide Web electronic publication, www.cdc. gov/nchs/data/nhanes/nhanes_03_04/ nhanes_analytic_guidelines_dec_2005.pdf.

CDC (CEnters For Disease Control) 2014: Questionnaires, Datasets, and Related Documentation: The National Health and Nutrition Examination Survey (Online). U.S. Department of Health and Human Services. World Wide Web electronic publication, www.cdc.gov/nchs/nhanes/nhanes_questionnaires.htm, 02/2014.

Cetinkaya Z., Yazar S., Gecici O., Namli M.N. 2007: AntiToxoplasma gondii antibodies in patients with schizophrenia - preliminary findings in a Turkish sample. Schizophr. Bull. 33: 789-791.
Dubey J.P. 1998: Advances in the life cycle of Toxoplasma gondii. Int. J. Parasitol. 28: 1019-1024.

Etkin A., WAGER T.D. 2007: Functional neuroimaging of anxiety: a meta-analysis of emotional processing in PTSD, social anxiety disorder, and specific phobia. Am. J. Psychiatry 164: 1476-1488.

Fekadu A., Shibre T., Cleare A.J. 2010: Toxoplasmosis as a cause for behaviour disorders - overview of evidence and mechanisms. Folia Parasitol. 57: 105-113.

FLEGR J. 2010: Influence of latent toxoplasmosis on the phenotype of intermediate hosts. Folia Parasitol. 57: 81-87.

FLEGR J. 2013: Influence of latent Toxoplasma infection on human personality, physiology and morphology: pros and cons of the Toxoplasma-human model in studying the manipulation hypothesis. J. Exp. Biol. 216: 127-133.

Hamdani N., Daban-Huard C., Lajnef M., Richard J.R., Delavest M., Godin O., Guen E.L., Vederine F.E., Lepine J.P., Jamain S., Houenou J., Corvoisier P.L., Aoki M., Moins-Teisserenc H., Charron D., Krishnamoorthy R., Yolken R., Dickerson F., Tamouza R., Leboyer M. 2013: Relationship between Toxoplasma gondii infection and bipolar disorder in a French sample. J. Affect. Disord. 148: 444-448.

Hamidinejat H., Ghorbanpoor M., Hosseini H., Alavi S.M., Nabavi L., Jalali M.H., Borojeni M.P., Jafari H., MoHAMmadaligol S. 2010: Toxoplasma gondii infection in firstepisode and inpatient individuals with schizophrenia. Int. J. Infect. Dis. 14: e978-981. 
Helzer J.E., Robins L.N. 1988: The diagnostic interview schedule: its development, evolution, and use. Soc. Psychiatry Psychiatr. Epidemiol. 23: 6-16.

Henriquez S.A., Brett R., Alexander J., Pratt J., Roberts C.W. 2009: Neuropsychiatric disease and Toxoplasma gondii infection. Neuroimmunomodulation 16: 122-133.

Hinze-Selch D., Daubener W., Eggert L., Erdag S., Stoltenberg R., Wilms S. 2007: A controlled prospective study of Toxoplasma gondii infection in individuals with schizophrenia: beyond seroprevalence. Schizophr. Bull. 33: 782-788.

Jones J.L., Dargelas V., Roberts J., Press C., Remington J.S., Montoya J.G. 2009: Risk factors for Toxoplasma gondii infection in the United States. Clin. Infect. Dis. 49: 878-884.

Jones J.L., Kruszon-Moran D., Wilson M. 2003: Toxoplasma gondii infection in the United States, 1999-2000. Emerg. Infect. Dis. 9: 1371-1374.

Jones J.L., Kruszon-Moran D., Wilson M., Mcquillan G., Navin T., Mcauley J.B. 2001: Toxoplasma gondii infection in the United States: seroprevalence and risk factors. Am. J. Epidemiol. 154: 357-365.

Jones-BRAndo L. 2003: Drugs used in the treatment of schizophrenia and bipolar disorder inhibit the replication of Toxoplasma gondii. Schizophr. Res. 62: 237-244.

Kessler R.C., Petukhova M., Sampson N.A., Zaslavsky A.M., Wittchen H.U. 2012: Twelve-month and lifetime prevalence and lifetime morbid risk of anxiety and mood disorders in the United States. Int. J. Methods Psychiatr. Res. 21: 169-184.

Montoya J.G., Liesenfeld O. 2004: Toxoplasmosis. Lancet 363: 1965-1976.

Novotná M., Hanusová J., Klose J., Preiss M., Havlíček J., Roubalová K., Flegr J. 2005: Probable neuroimmunological link between Toxoplasma and cytomegalovirus infections and personality changes in the human host. BMC Infect. Dis. 5: 54.

Pearce B.D., Kruszon-Moran D., Jones J.L. 2012: The relationship between Toxoplasma gondii infection and mood disorders in the third National Health and Nutrition Survey. Biol. Psychiatry. 72: 290-295.

Pedersen M.G., Mortensen P.B., Norgaard-Pedersen B., Postolache T.T. 2012: Toxoplasma gondii infection and self-directed violence in mothers. Arch. Gen. Psychiatry 69: $1123-1130$.

Prandovszky E., Gaskell E., Martin H., Dubey J.P., WebSter J.P., Mcconkey G.A. 2011: The neurotropic parasite Toxoplasma gondii increases dopamine metabolism. PLoS ONE 6: e23866.

Received 10 September 2013
Skallová A., Novotná M., Kolbeková P., Gasová Z., Veselý V., Sechovská M., Flegr J. 2005: Decreased level of novelty seeking in blood donors infected with Toxoplasma. Neuroendocrinol. Lett. 26: 480-486.

Stibbs H.H. 1985: Changes in brain concentrations of catecholamines and indoleamines in Toxoplasma gondii infected mice. Ann. Trop. Med. Parasitol. 79: 153-157.

Torrey E.F., Bartko J.J., Lun Z.R., Yolken R.H. 2007: Antibodies to Toxoplasma gondii in patients with schizophrenia: a meta-analysis. Schizophr. Bull. 33: 729-736.

Tromp D.P., Grupe D.W., Oathes D.J., Mcfarlin D.R., Hernandez P.J., Kral T.R., Lee J.E., Adams M., Alexander A.L., NitsChKe J.B. 2012: Reduced structural connectivity of a major frontolimbic pathway in generalized anxiety disorder. Arch. Gen. Psychiatry 69: 925-934.

Vasa R.A., Grados M., Slomine B., Herskovits E.H., Thompson R.E., Salorio C., Christensen J., Wursta C., Riddle M.A., GerRing J.P. 2004: Neuroimaging correlates of anxiety after pediatric traumatic brain injury. Biol. Psychiatry. 55: 208-216.

Vyas A., Kim S.K., Giacomini N., Boothroyd J.C., SAPOlsky R.M. 2007: Behavioral changes induced by Toxoplasma infection of rodents are highly specific to aversion of cat odors. Proc. Natl. Acad. Sci. USA 104: 6442-6447.

Wang H.L., Wang G.H., Li Q.Y., Shu C., Jiang M.S., Guo Y. 2006: Prevalence of Toxoplasma infection in first-episode schizophrenia and comparison between Toxoplasma-seropositive and Toxoplasma-seronegative schizophrenia. Acta. Psychiatr. Scand. 114: 40-48.

Webster J.P. 2007: The effect of Toxoplasma gondii on animal behavior: playing cat and mouse. Schizophr. Bull. 33: 752-756.

Webster J.P., Lamberton P.H., Donnelly C.A., Torrey E.F. 2006: Parasites as causative agents of human affective disorders? The impact of anti-psychotic, mood-stabilizer and antiparasite medication on Toxoplasma gondii's ability to alter host behaviour. Proc. Biol. Sci. 273: 1023-1030.

Xiao J., Kannan G., Jones-Brando L., Brannock C., Krasnova I.N., Cadet J.L., Pletnikov M., Yolken R.H. 2012: Sex-specific changes in gene expression and behavior induced by chronic Toxoplasma infection in mice. Neuroscience 206: $39-48$

Zhang Y., Traskman-Bendz L., Janelidze S., Langenberg P., Saleh A., Constantine N., Okusaga O., Bay-Richter C., Brundin L., Postolache T.T. 2012: Toxoplasma gondii immunoglobulin $\mathrm{G}$ antibodies and nonfatal suicidal self-directed violence. J. Clin. Psychiatry 73: 1069-1076. 